\title{
Model-independent Distance Calibration and Curvature Measurement Using Quasars and Cosmic Chronometers
}

\author{
Jun-Jie Wei ${ }^{1,2,3}$ (1D) and Fulvio Melia ${ }^{4,5}$ (D) \\ ${ }^{1}$ Purple Mountain Observatory, Chinese Academy of Sciences, Nanjing 210033, People's Republic of China; jjwei@pmo.ac.cn \\ ${ }^{2}$ Guangxi Key Laboratory for Relativistic Astrophysics, Guangxi University, Nanning 530004, People's Republic of China \\ ${ }^{3}$ University of Chinese Academy of Sciences, Beijing 100049, People's Republic of China \\ ${ }^{4}$ Department of Physics, The Applied Math Program, and Department of Astronomy, The University of Arizona, Tucson, AZ 85721, USA; fmelia@email.arizona.edu \\ Received 2019 September 26; revised 2019 November 30; accepted 2019 December 2; published 2020 January 14
}

\begin{abstract}
We present a new model-independent method to determine spatial curvature and to mitigate the circularity problem affecting the use of quasars as distance indicators. Cosmic-chronometer measurements are used to construct the curvature-dependent luminosity distance $D_{L}^{\text {cal }}\left(\Omega_{K}, z\right)$ using a polynomial fit. Based on the reconstructed $D_{L}^{\text {cal }}\left(\Omega_{K}, z\right)$ and the known ultraviolet versus X-ray luminosity correlation of quasars, we simultaneously place limits on the curvature parameter $\Omega_{K}$ and the parameters characterizing the luminosity correlation function. This model-independent analysis suggests that a mildly closed universe $\left(\Omega_{K}=-0.918 \pm 0.429\right)$ is preferred at the $2.1 \sigma$ level. With the calibrated luminosity correlation, we build a new data set consisting of 1598 quasar distance moduli, and use these calibrated measurements to test and compare the standard $\Lambda \mathrm{CDM}$ model and the $R_{\mathrm{h}}=c t$ universe. Both models account for the data very well, though the optimized flat $\Lambda \mathrm{CDM}$ model has one more free parameter than $R_{\mathrm{h}}=c t$, and is penalized more heavily by the Bayes Information Criterion. We find that $R_{\mathrm{h}}=c t$ is slightly favored over $\Lambda \mathrm{CDM}$ with a likelihood of $\sim 57.7 \%$ versus $42.3 \%$.
\end{abstract}

Unified Astronomy Thesaurus concepts: Quasars (1319); Cosmological parameters (339); Cosmological models (337); Distance indicators (394); Observational cosmology (1146)

\section{Introduction}

Quasars are the most luminous persistent sources in the universe, detected up to redshifts $z \sim 7.5$ (Mortlock et al. 2011; Wu et al. 2015; Bañados et al. 2018). It is generally accepted that ultraviolet (UV) photons of active galactic nuclei are emitted by an accretion disk, while X-rays are Compton upscattered photons from a hot corona above the disk. A nonlinear relation between the UV and X-ray monochromatic luminosities of quasars has been known for over three decades (Avni \& Tananbaum 1986), but only recently has the uncomfortably large dispersion in the correlation been mitigated by refining the selection criteria and flux measurements (Risaliti \& Lusso 2015, 2019; Lusso \& Risaliti 2016, 2017). This offers the possibility of using quasars as a complementary cosmic probe at high $z$. Some cosmological constraints have been obtained based on this refined correlation (Risaliti \& Lusso 2015, 2019; López-Corredoira et al. 2016; Khadka \& Ratra 2019; Lusso et al. 2019; Melia 2019). There is a circularity problem when treating high- $z$ quasars as relative standard candles, however. The problem arises from the fact that, given the lack of very low- $z$ quasars, the correlation between the X-ray and UV luminosities must be established assuming a background cosmology. All of the previous applications of the luminosity correlation attempting to overcome the circularity problem have used simultaneous multiparameter fitting in the context of specifically selected models. One of the principal limitations of this approach is that none of the chosen models may actually be the true cosmology.

These hurdles notwithstanding, there is significant motivation to use these data for cosmological studies because high- $z$ quasars extend our reach well beyond other kinds of sources,

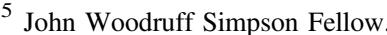

such as Type Ia supernovae ( $\mathrm{SNe}$ Ia), which may be seen only as far as $z \sim 1.8$. Quasars may therefore help to shed light on one of the most pressing issues in modern cosmology, i.e., the spatial curvature of the universe. Estimating whether the universe is open, flat, or closed is crucial for us to understand its evolution and the nature of dark energy (Ichikawa et al. 2006; Clarkson et al. 2007; Gong \& Wang 2007; Virey et al. 2008). Any significant deviation from zero spatial curvature would have a profound impact on the inflationary paradigm and its underlying physics (Eisenstein et al. 2005; Tegmark et al. 2006; Wright 2007; Zhao et al. 2007).

Current cosmological observations strongly favor a spatially flat universe, e.g., the combined Planck 2018 cosmic microwave background (CMB) and baryon acoustic oscillation measurements, which suggest that $\Omega_{K}=0.001 \pm 0.002$ (Planck Collaboration et al. 2018). ${ }^{6}$ These constraints, however, are based on the pre-assumption of a specific cosmological model (e.g., the standard $\Lambda$ CDM model). Because of the strong degeneracy between spatial curvature and the equation of state of dark energy, it is difficult to constrain the two quantities simultaneously. In general, dark energy is assumed to be a cosmological constant for the estimation of curvature or, conversely, the universe is assumed to be flat in a dark energy analysis. But a simple flatness assumption may result in an incorrect reconstruction of the dark energy equation of state, even if the real curvature is tiny (Clarkson et al. 2007), and a cosmological constant assumption may lead to confusion between $\Lambda \mathrm{CDM}$ and a dynamical dark energy model (Virey et al. 2008). Therefore, it would be better to measure the

\footnotetext{
6 The combination of the Planck 2018 CMB temperature and polarization power spectra data slightly favor a mildly closed universe, i.e., $\Omega_{K}=-0.044_{-0.015}^{+0.018}$ (Planck Collaboration et al. 2018). Other recent works showed that the Planck 2015 CMB anisotropy data also favor a mildly closed universe (see Ooba et al 2018a, 2018b and references therein).
} 
curvature parameter by purely geometrical and model-independent methods. A non-exhaustive list of previous works attempting to measure spatial curvature in a model-independent way includes Bernstein (2006), Clarkson et al. (2007), Shafieloo \& Clarkson (2010), Mortsell \& Jonsson (2011), Li et al. (2014, 2016, 2018), Sapone et al. (2014), Räsänen et al. (2015), Cai et al. (2016) ,Yu \& Wang (2016), L'Huillier \& Shafieloo (2017); Liao et al. (2017), Rana et al. (2017), Wang et al. (2017), Wei \& Wu (2017), Xia et al. (2017), Denissenya et al. (2018), Wei (2018), Yu et al. (2018), Cao et al. (2019), Ruan et al. (2019), and Qi et al. (2019).

In this paper, we propose a new model-independent method to simultaneously calibrate the UV versus X-ray luminosity correlation for quasars and to determine the curvature of the universe. Using a polynomial fitting technique (Amati et al. 2019), one can reconstruct a continuous $H(z)$ function representing the expansion rate measurements using cosmic chronometers without the pre-assumption of any particular cosmological model. The co-moving distance function can then be directly derived by integrating the reconstructed $H(z)$ function. Then, with the curvature parameter $\Omega_{K}$ taken into consideration, the co-moving distance can be transformed into the curvature-dependent luminosity distance $D_{L}^{\text {cal }}\left(\Omega_{K}, z\right)$. Finally, by combining $D_{L}^{\text {cal }}\left(\Omega_{K}, z\right)$ with the redshift and flux measurements of quasars, we obtain model-independent constraints on the spatial curvature and the parameters characterizing the quasar luminosity relation. Refining their selection technique to avoid the inclusion of possible contaminants, Risaliti \& Lusso (2019) obtained a final catalog of 1598 quasars with reliable measurements of both the intrinsic X-ray and UV emissions. We adopt this high-quality quasar sample covering the redshift range $0.04<z<5.1$ for the analysis reported in this paper.

The rest of the paper is organized as follows. In Section 2, we introduce the model-independent method used to calibrate the quasar luminosity relation and determine the curvature parameter. The calibrated luminosity relation is then used to test different cosmological models. The outcome of our model comparison will be described in Section 3, followed by our conclusions in Section 4.

\section{Methodology}

\subsection{Curvature-dependent Distance from Cosmic-chronometer Measurements}

The expansion rate of the universe, $H(z) \equiv \dot{a} / a$, where $a(t)=1 /(1+z)$, can be obtained directly from the redshift-time derivative $d z / d t$ using $H(z)=-\frac{1}{1+z} \frac{d z}{d t}$ at any redshift $z \neq 0$. For this purpose, the differential age evolution of passively evolving galaxies can be used to measure the expansion rate $H(z)$ in a cosmology-independent way (Jimenez \& Loeb 2002). These galaxies are commonly referred to as "cosmic chronometers." The most recent sample of 31 cosmic-chronometer measurements (see Wei 2018 and references therein) is shown in Figure 1(a). To avoid the circularity problem, Amati et al. (2019) proposed a model-independent technique to reconstruct a reasonable $H(z)$ function that best approximates the discrete cosmic-chronometer data. Following these authors, we fit the $H(z)$ measurements employing a Bézier parametric curve of degree $n$ :

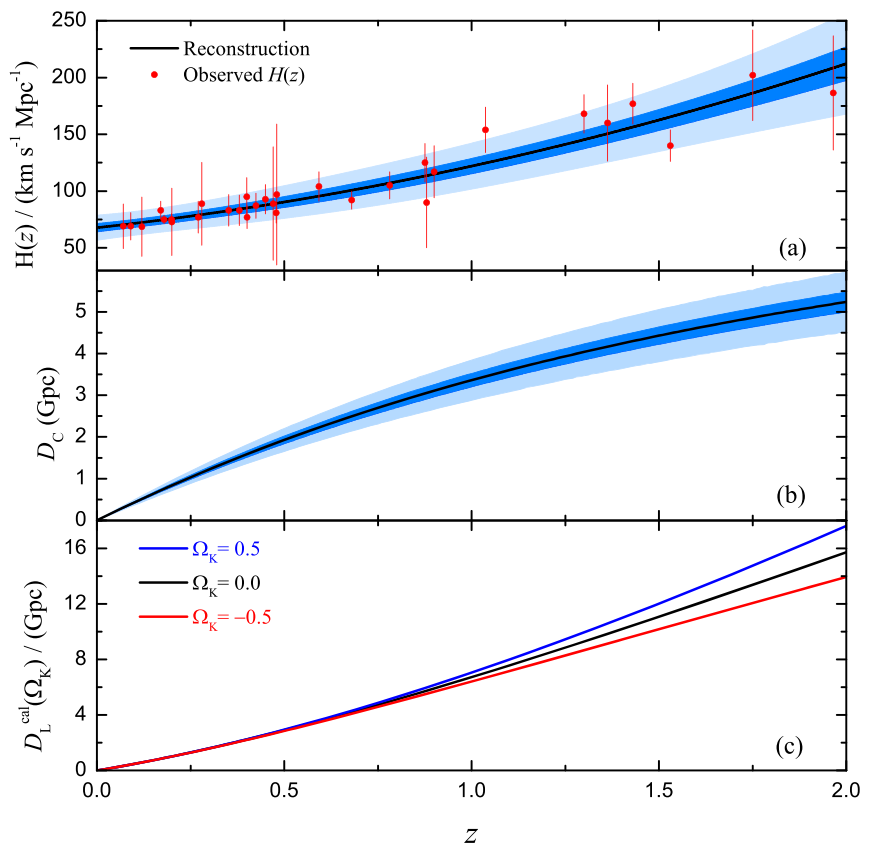

Figure 1. Panel (a): measured expansion rates from cosmic-chronometer measurements (solid points with the vertical error bars) and the reconstructed Hubble parameter function $H(z)$ (solid line) from the data. Panel (b): reconstructed co-moving distance function $D_{C}(z)$ (solid line). The shaded areas in both panels (a) and (b) represent the $1 \sigma$ and $3 \sigma$ confidence regions of the corresponding reconstructions. Panel (c): indicative dependence of the luminosity distance $D_{L}^{\text {cal }}$ derived from the $D_{C}(z)$ function on the spatial curvature parameter $\Omega_{K}$.

$$
\begin{aligned}
& H_{n}(z)=\sum_{d=0}^{n} \beta_{d} h_{n}^{d}(z) \\
& h_{n}^{d}(z) \equiv \frac{n !\left(z / z_{\mathrm{m}}\right)^{d}}{d !(n-d) !}\left(1-\frac{z}{z_{\mathrm{m}}}\right)^{n-d},
\end{aligned}
$$

where $z_{\mathrm{m}}$ is the maximum redshift of the cosmic-chronometer data set and the $\beta_{d}$ are positive coefficients of the linear combination of Bernstein basis polynomials $h_{n}^{d}(z)$ in the range $0 \leqslant z \leqslant z_{\mathrm{m}}$. For $z=0$ and $d=0$, it is easy to identify $\beta_{0} \equiv H_{0}$. Since the high value of $n(n>2)$ would lead to an oscillatory behavior of the approximating function, we adopt $n=2$ in fitting the discrete $H(z)$ data (Amati et al. 2019). The reconstructed $H(z)$ function (solid line) with $1 \sigma$ and $3 \sigma$ confidence regions (shaded areas) is plotted in Figure 1(a). The best-fitting parameters are $H_{0}=$ $67.76 \pm 3.68, \beta_{1}=103.33 \pm 11.16$, and $\beta_{2}=208.45 \pm 14.29$ (all in units of $\mathrm{km} \mathrm{s}^{-1} \mathrm{Mpc}^{-1}$ ). The Hubble constant $H_{0}$ obtained here is in good agreement with the value inferred from Planck $\left(H_{0}=67.4 \pm 0.5 \mathrm{~km} \mathrm{~s}^{-1} \mathrm{Mpc}^{-1}\right.$; Planck Collaboration et al. 2018), and is also compatible at the $1.6 \sigma$ level with the estimate based on local distance measurements $\left(H_{0}=74.03 \pm 1.42 \mathrm{~km} \mathrm{~s}^{-1}\right.$ $\mathrm{Mpc}^{-1}$; Riess et al. 2019). For consistency, we use this best-fit value of $H_{0}$ for the distance estimations in the following analysis.

The line-of-sight co-moving distance

$$
D_{C}(z)=c \int_{0}^{z} \frac{d z^{\prime}}{H_{2}\left(z^{\prime}\right)}
$$

(Hogg 1999) can then be derived by integrating the $H_{2}(z)$ function with respect to redshift (solid line in Figure 1(b)). Since the error propagation is complicated, we estimate 
uncertainties in $D_{C}(z)$ based on 10,000 Monte Carlo simulations utilizing the uncertainties in the coefficients $H_{0}, \beta_{1}$, and $\beta_{2}$. The shaded areas in Figure 1(b) represent the $1 \sigma$ and $3 \sigma$ uncertainties taking into account the spread of all the Monte Carlo simulation results.

With the reconstructed co-moving distance function $D_{C}(z)$, as well as its $1 \sigma$ uncertainty $\sigma_{D_{C}}$, the curvature-dependent luminosity distance $D_{L}^{\text {cal }}$ can then be expressed as

$$
\frac{D_{L}^{\text {cal }}\left(\Omega_{K}, z\right)}{(1+z)}= \begin{cases}\frac{D_{H}}{\sqrt{\Omega_{K}}} \sinh \left[\sqrt{\Omega_{K}} \frac{D_{C}(z)}{D_{H}}\right] & \text { for } \Omega_{K}>0 \\ D_{C}(z) & \text { for } \Omega_{K}=0 \\ \frac{D_{H}}{\sqrt{\left|\Omega_{K}\right|}} \sin \left[\sqrt{\left|\Omega_{K}\right|} \frac{D_{C}(z)}{D_{H}}\right] & \text { for } \Omega_{K}<0\end{cases}
$$

with its corresponding uncertainty

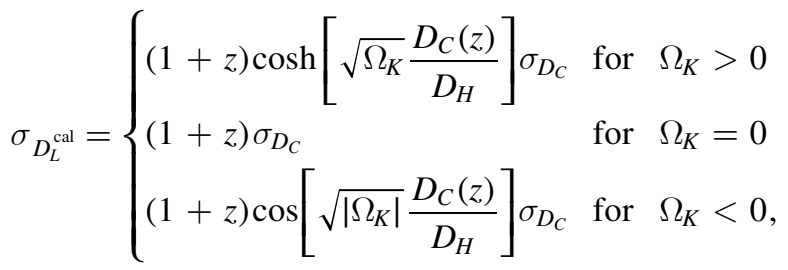

where $D_{H}=c / H_{0}$ is the Hubble distance. In Figure 1(c), we illustrate the dependence of $D_{L}^{\text {cal }}$ (derived from cosmicchronometer measurements) on the spatial curvature $\Omega_{K}$.

\subsection{Distance Calibration and Curvature Measurement}

We are now in position to use $D_{L}^{\text {cal }}\left(\Omega_{K}, z\right)$ derived from cosmic-chronometer measurements to calibrate the nonlinear relation between the UV and X-ray luminosities of quasars, which is commonly written using the following ansatz:

$$
\log _{10} L_{\mathrm{X}}^{\mathrm{cal}}=\gamma \log _{10} L_{\mathrm{UV}}^{\mathrm{cal}}+\kappa .
$$

We re-write this to bring out its explicit dependence on the luminosity distance:

$$
\begin{aligned}
\log _{10} F_{\mathrm{X}} & =\Phi\left(F_{\mathrm{UV}}, D_{L}^{\text {cal }}\left(\Omega_{K}, z\right)\right. \\
& =\kappa^{\prime}+\gamma \log _{10} F_{\mathrm{UV}}+2(\gamma-1) \log _{10} D_{L}^{\text {cal }},
\end{aligned}
$$

where $F_{\mathrm{X}}$ and $F_{\mathrm{UV}}$ are the rest-frame X-ray and UV fluxes, respectively, and $\kappa^{\prime}$ is a constant that contains the slope $\gamma$ and intercept $\kappa$ in Equation (5), i.e., $\kappa^{\prime}=\kappa+(\gamma-1) \log _{10} 4 \pi$.

One of the limitations we must deal with in using the quasar data, however, is that the $H(z)$ measurements extend only to $z=2$. As such, we shall employ only a sub-set of the entire quasar sample that overlaps with the $H(z)$ catalog for the calibration. The calibration of the luminosity relation will therefore be based only on the 1330 quasars at $z<2$. The calibrated luminosity relation, along with the curvature parameter $\Omega_{K}$, can be fitted by maximizing the likelihood

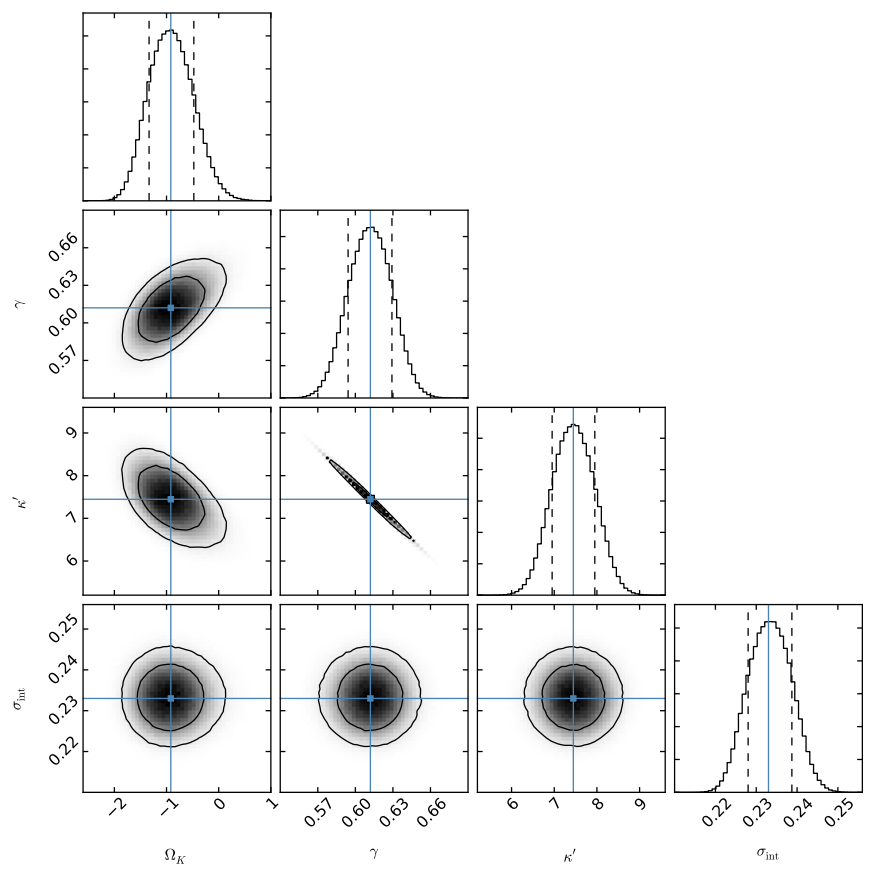

Figure 2. 1D marginalized probability distributions and 2D regions with the $1-2 \sigma$ contours corresponding to the cosmic curvature $\Omega_{K}$ and the parameters $\left(\gamma, \kappa^{\prime}\right.$, and $\left.\sigma_{\text {int }}\right)$ characterizing the luminosity relation. The vertical solid lines represent the best fits, and the vertical dashed lines enclose the $1 \sigma$ credible region.

function:

$$
\begin{aligned}
\mathcal{L}= & \prod_{i}^{1330} \frac{1}{\sqrt{2 \pi} \sigma_{\mathrm{tot}, i}} \\
& \times \exp \left\{-\frac{\left[\log _{10}\left(F_{\mathrm{X}}\right)_{i}-\Phi\left(\left[F_{\mathrm{UV}}\right]_{i}, D_{L}^{\mathrm{cal}}\left[\Omega_{K}, z_{i}\right]\right)\right]^{2}}{2 \sigma_{\mathrm{tot}, i}^{2}}\right\},
\end{aligned}
$$

where $\Phi\left(\left[F_{\mathrm{UV}}\right]_{i}, D_{L}^{\text {cal }}\left[\Omega_{K}, z_{i}\right]\right)$ is given by Equation (6) and the variance

$$
\sigma_{\text {tot }}^{2}=\sigma_{\text {int }}^{2}+\sigma_{\log _{10} F_{\mathrm{X}}, i}^{2}+\left[2(\gamma-1) \frac{\sigma_{D_{L}^{\text {cal }}, i}}{\ln 10 D_{L}^{\text {cal }}\left(\Omega_{K}, z_{i}\right)}\right]^{2}
$$

is given in terms of the global intrinsic dispersion $\sigma_{\text {int }}$, the measurement uncertainty $\sigma_{\log _{10} F_{\mathrm{X}}, i}$ in $\log _{10}\left(F_{\mathrm{X}}\right)_{i}$, and the propagated uncertainty of $D_{L}^{\text {cal }}\left(\Omega_{K}, z_{i}\right)$ derived from cosmicchronometer measurements. The uncertainty in $\log _{10}\left(F_{\mathrm{UV}}\right)_{i}$ is presumed to be insignificant compared to the three terms in Equation (8), and is therefore ignored in our calculations. In this case, the free parameters are $\sigma_{\text {int }}, \gamma, \kappa^{\prime}$, and the curvature parameter $\Omega_{K}$. We use the Python Markov chain Monte Carlo module EMCEE (Foreman-Mackey et al. 2013), to get the best-fitting values and their corresponding uncertainties for these parameters by generating sample points of the probability distribution. The 1D marginalized probability distribution for each free parameter and 2D plots of the 1-2 $\sigma$ confidence regions for two-parameter combinations are displayed in Figure 2. These contours show that at the $1 \sigma$ level, the optimized parameter 
values are $\Omega_{K}=-0.918 \pm 0.429, \gamma=0.612 \pm 0.017, \kappa^{\prime}=$ $7.447 \pm 0.500$, and $\sigma_{\text {int }}=0.233 \pm 0.005$. We find that the measured $\Omega_{K}$ deviates slightly from zero spatial curvature, implying that the current quasar data favor a mildly closed universe with a $2.1 \sigma$ degree of confidence.

Given the potential impact of such a result, we next consider whether the reconstruction scheme affects the curvature measurement. We have also performed a parallel comparative analysis of the discrete cosmic-chronometer data using a different approach, based on the so-called Padé approximation.

The Padé approximation to the $H(z)$ function is described by the rational polynomial (of a specified order)

$$
H_{m n}(z) \equiv \frac{\sum_{i=0}^{m} a_{i} z^{i}}{1+\sum_{i=1}^{n} b_{i} z^{i}},
$$

where the two non-negative integers ( $m$ and $n$ ) represent the degrees of the numerator and the denominator, respectively. The coefficients $a_{i}$ and $b_{i}$ can be determined by fitting $H_{m n}(z)$ to the discrete $H(z)$ data. For $z=0$, it is easy to identify $a_{0} \equiv H_{0}$. There are three free parameters in the Bézier polynomial reconstruction. To keep the number of free parameters the same, we have three different Padé approximations of order $(m=2, n=0),(m=0, n=2)$, and $(m=1, n=1)$. That is, $H_{20}(z)=a_{0}+a_{1} z+a_{2} z^{2}, \quad H_{02}(z)=a_{0}\left[1+b_{1} z+b_{2} z^{2}\right]^{-1}$, and $H_{11}(z)=\left[a_{0}+a_{1} z\right]\left[1+b_{1} z\right]^{-1}$.

The Padé approximations with $n=0$ actually reduce to Taylor polynomials. We find that $H_{20}(z)$ fits the data with a reduced $\chi_{\text {dof }}^{2}=14.74 / 28=0.53$, while $H_{02}(z)$ and $H_{11}(z)$ fit the data with an unsatisfactory $\chi_{\text {dof }}^{2}=360.84 / 28=12.89$ and $\chi_{\text {dof }}^{2}=122.87 / 28=4.39$, respectively. Therefore, we shall consider only the Padé approximation of order $(2,0)$ for our comparison. The best-fitting parameters of the reconstructed $H_{20}(z)$ function are $a_{0}=67.76 \pm 3.69, a_{1}=36.20 \pm 14.57$, and $a_{2}=18.01 \pm 9.53$ (all in units of $\mathrm{km} \mathrm{s}^{-1} \mathrm{Mpc}^{-1}$ ). The subsequent steps to calibrate the distance and measure the curvature are then the same as described above.

Using the Padé-based reconstruction, the constraints on the cosmic curvature and the parameters of the luminosity relation are $\Omega_{K}=-0.930 \pm 0.430, \gamma=0.612 \pm 0.017, \kappa^{\prime}=$ $7.452 \pm 0.501$, and $\sigma_{\text {int }}=0.233 \pm 0.005$. Comparing this inferred $\Omega_{K}$ with that obtained using the Bézier polynomial reconstruction $\left(\Omega_{K}=-0.918 \pm 0.429\right)$, we see that the adoption of a different reconstruction scheme has only a minimal influence on the results. For the rest of this paper, we shall therefore adopt the calibrated results based on the Bézier polynomial reconstruction.

The distribution of logarithmic X-ray luminosities, $\log _{10} L_{\mathrm{X}}^{\text {cal }}$, versus the UV luminosities, $\log _{10} L_{\mathrm{UV}}^{\text {cal }}$, is shown in Figure 3 for the 1330 quasars at $z<2$, together with the best-fitting line. The propagated uncertainties of $\log _{10} L_{\mathrm{X}}^{\text {cal }}$ and $\log _{10} L_{\mathrm{UV}}^{\text {cal }}$ are calculated from

$$
\sigma_{\log _{10} L_{\mathrm{X}}^{\text {cal }}}=\left[\sigma_{\log _{10} F_{\mathrm{X}}}^{2}+\left(2 \frac{\sigma_{D_{L}^{\text {cal }}}}{\ln 10 D_{L}^{\text {cal }}}\right)^{2}\right]^{1 / 2}
$$

and

$$
\sigma_{\log _{10} L \text { cal }}=2 \frac{\sigma_{D_{L}^{\text {cal }}}^{\text {cal }}}{\ln 10 D_{L}^{\text {cal }}},
$$

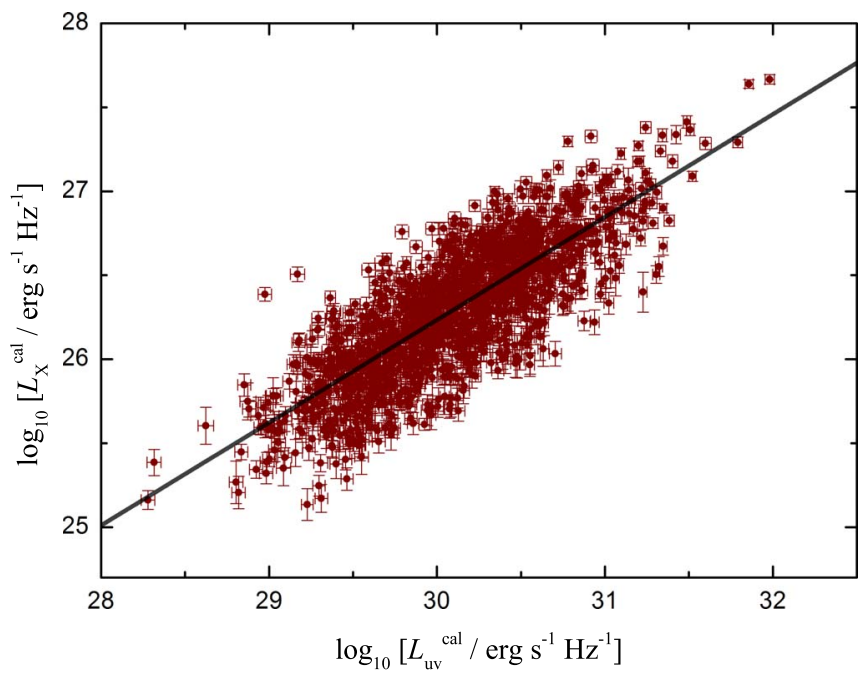

Figure 3. Calibrated distribution in the $L_{\mathrm{UV}}^{\text {cal }}-L_{\mathrm{X}}^{\text {cal }}$ plane for 1330 quasars at $z<2$. The solid line shows the best-fit result.

respectively.

Risaliti \& Lusso (2015) found no evidence of a redshift evolution for the luminosity relation. We shall therefore assume that the optimized relation we have derived at $z<2$ holds at all redshifts with the same slope and intercept. Extrapolating the calibrated luminosity relation to high $z(z>2)$, we then derive the distance moduli of the whole quasar sample, including 1598 sources. With the calibrated luminosity relation, the distance modulus of a quasar can be obtained using

$$
\mu_{\mathrm{obs}}=\frac{5}{2(\gamma-1)}\left[\log _{10} F_{\mathrm{X}}-\gamma \log _{10} F_{\mathrm{UV}}-\kappa^{\prime}\right]-97.447 .
$$

The error $\sigma_{\mu_{\mathrm{obs}}}$ in $\mu_{\mathrm{obs}}$ is calculated via error propagation, i.e.,

$$
\begin{aligned}
\sigma_{\mu_{\mathrm{obs}}}= & \mu_{\mathrm{obs}}\left[\left(\frac{\sigma_{\gamma}}{\gamma-1}\right)^{2}\right. \\
& \left.+\left(\frac{\sigma_{y}}{\log _{10} F_{\mathrm{X}}-\gamma \log _{10} F_{\mathrm{UV}}-\kappa^{\prime}}\right)^{2}\right]^{1 / 2},
\end{aligned}
$$

where $\sigma_{y}=\left[\sigma_{\log _{10} F_{\mathrm{X}}}^{2}+\left(\sigma_{\gamma} \log _{10} F_{\mathrm{UV}}\right)^{2}+\sigma_{\kappa^{\prime}}^{2}+\sigma_{\text {int }}^{2}\right]^{1 / 2}$, and $\sigma_{\gamma}$ and $\sigma_{\kappa^{\prime}}$ are the uncertainties of the slope $\gamma$ and intercept $\kappa^{\prime}$. The calibrated quasar Hubble diagram is shown in Figure 4.

\section{Testing Cosmological Models}

In this section, we use the calibrated quasar distance moduli to test certain cosmological models. The cosmological parameters are optimized by minimizing the $\chi^{2}$ statistic, i.e.,

$$
\chi^{2}=\sum_{i}^{1598} \frac{\left[\mu_{\mathrm{obs}, i}-\mu_{\mathrm{th}}\left(z_{i}\right)\right]^{2}}{\sigma_{\mu_{\mathrm{obs},}, i}^{2}},
$$

where $\mu_{\text {th }} \equiv 5 \log _{10}\left[D_{L}(z) / \mathrm{Mpc}\right]+25$ is the theoretical distance modulus of a quasar at redshift $z$. The determination of $D_{L}$ requires the assumption of a particular cosmological model. For the sake of consistency, we adopt the Hubble constant as the best-fitting value derived from the model-independent analysis of the cosmic-chronometer data $\left(H_{0}=67.76 \mathrm{~km} \mathrm{~s}^{-1}\right.$ 


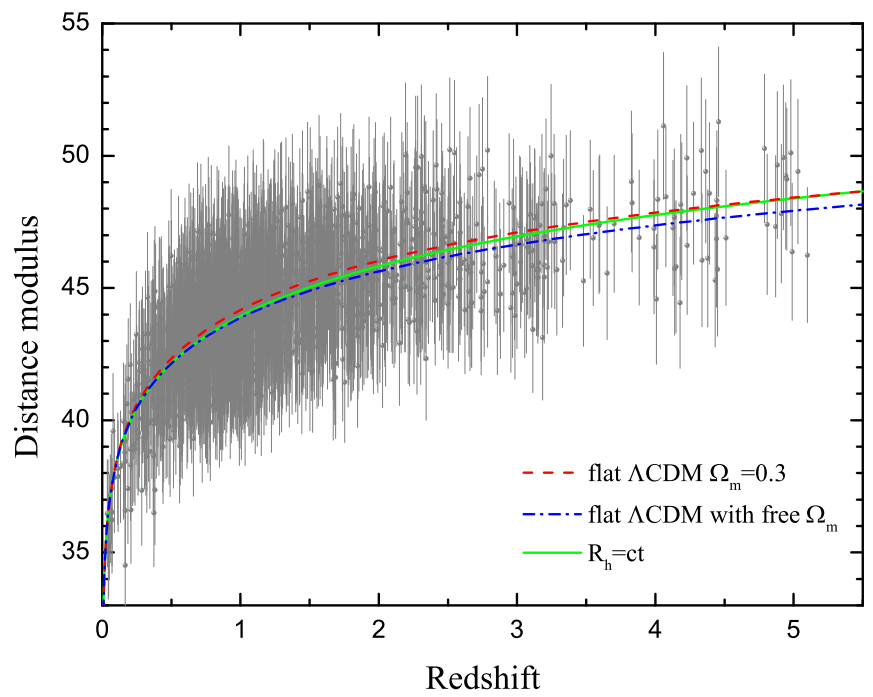

Figure 4. Hubble diagram for 1598 calibrated quasars (points), with $1 \sigma$ uncertainties. The theoretical curves show the concordance flat $\Lambda$ CDM model with fixed $\Omega_{\mathrm{m}}=0.3$ (dashed), the optimized flat $\Lambda \mathrm{CDM}$ model with $\Omega_{\mathrm{m}}=0.582_{-0.059}^{+0.074}$ (dotted-dashed), and the $R_{\mathrm{h}}=c t$ universe (solid).

$\mathrm{Mpc}^{-1}$ ) in the optimization procedure. Here we discuss how the fits have been optimized for $\Lambda \mathrm{CDM}$ and $R_{\mathrm{h}}=c t$. The outcome for each model is carefully described and discussed in subsequent sections.

\section{1. $\Lambda C D M$}

In a flat $\Lambda \mathrm{CDM}$ universe with zero spatial curvature, the theoretical luminosity distance is given as

$$
D_{L}^{\Lambda \mathrm{CDM}}(z)=\frac{c}{H_{0}}(1+z) \int_{0}^{z} \frac{d z^{\prime}}{\sqrt{\Omega_{\mathrm{m}}\left(1+z^{\prime}\right)^{3}+\Omega_{\Lambda}}},
$$

where $\Omega_{\mathrm{m}}$ is the scaled matter density today and $\Omega_{\Lambda}=1-\Omega_{\mathrm{m}}$ is the cosmological constant energy density. (We ignore the contribution from radiation, which is insignificant compared to that of matter and dark energy in this redshift range.) The Hubble constant $H_{0}$ is fixed to be the value obtained from the modelindependent analysis of the discrete $H(z)$ data, so the sole remaining parameter in flat $\Lambda \mathrm{CDM}$ is $\Omega_{\mathrm{m}}$. With the calibrated distance moduli of quasars, the resulting constraint on $\Omega_{\mathrm{m}}$ is shown in Figure 5. The best-fitting value is $\Omega_{\mathrm{m}}=0.582_{-0.059}^{+0.074}$ at the $1 \sigma$ confidence level. With $1598-1=1597$ degrees of freedom, the reduced $\chi^{2}$ is $\chi_{\text {dof }}^{2}=648.57 / 1597=0.41$.

This optimized $\Omega_{\mathrm{m}}$ value is, however, in some tension with that inferred from other kinds of data. In particular, the concordance $\Lambda \mathrm{CDM}$ model with $\Omega_{\mathrm{m}} \approx 0.3$ seems to account best for many other cosmological observations (Aubourg et al. 2015; Planck Collaboration et al. 2018; Scolnic et al. 2018). It must be emphasized, however, that the $\Lambda \mathrm{CDM}$ is rarely tested in the redshift range between the farthest observed SNe Ia and the last scattering surface (producing the CMB). Recently, Risaliti \& Lusso (2019) showed that a $\sim 4 \sigma$ tension exists between the high- $z$ data and the $\Lambda$ CDM model, based on a model-independent parameterization of the Hubble diagram using these sources. Fitting the calibrated distance moduli of 1598 quasars obtained from our model-independent technique with the concordance model using $\Omega_{\mathrm{m}}=0.3$, we obtain a $\chi^{2}$ per degree of freedom of $\chi_{\text {dof }}^{2}=676.60 / 1598=0.42$.

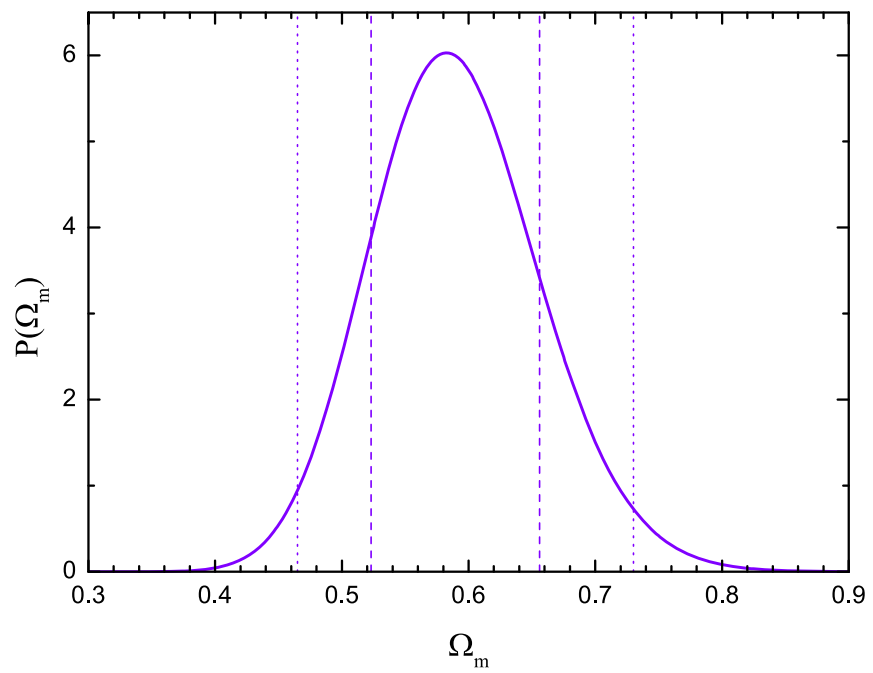

Figure 5. Probability distribution function of the matter density parameter $\Omega_{\mathrm{m}}$. The $1 \sigma$ and $2 \sigma$ credible intervals are indicated by the vertical dashed and dotted lines, respectively.

Table 1

Best-fitting Results in Different Cosmological Models with the Whole Sample of 1598 Quasars

\begin{tabular}{lcccc}
\hline \hline Model & $\Omega_{\mathrm{m}}$ & $\chi_{\text {dof }}^{2}$ & BIC & Likelihood \\
\hline Concordance & 0.3 (fixed) & 0.42 & 676.60 & $1 \mathrm{E}-3 \%$ \\
$\Lambda$ CDM & $0.582_{-0.059}^{+0.074}$ & 0.41 & 655.95 & $42.31 \%$ \\
$R_{\mathrm{h}}=c t$ & $\cdots$ & 0.41 & 655.33 & $57.69 \%$ \\
\hline
\end{tabular}

These results are somewhat consistent with those of Risaliti \& Lusso (2019), in the sense that the concordance model does not provide the best fit to these quasar data extending up to $z \sim 6$, when compared to other formulations and/or parameter values. Having said this, the errors reported for the data appear to be larger than one would expect from their scatter, which is probably why our inferred $\chi_{\text {dof }}^{2}$ 's are much smaller than 1 . Simply on the basis of the reduced $\chi^{2}$, the concordance model fits the data quite well. When compared to the other two fits reported in Table 1, however, one can see, especially from Figure 5, that the concordance model with $\Omega_{\mathrm{m}}=0.3$ is disfavored at the $4.3 \sigma$ level, somewhat confirming the result of Risaliti \& Lusso (2019).

\subsection{The $R_{\mathrm{h}}=$ ct Universe}

The luminosity distance in the $R_{\mathrm{h}}=c t$ universe (Melia 2003, 2007, 2013; Melia \& Shevchuk 2012; Wei et al. 2015), is given as

$$
D_{L}^{R_{\mathrm{h}}=c t}(z)=\frac{c}{H_{0}}(1+z) \ln (1+z) .
$$

The $R_{\mathrm{h}}=c t$ cosmology has only one free parameter, $H_{0}$, but since the Hubble constant is fixed, there are no free parameters left to fit the quasar Hubble diagram. The $\chi^{2}$ per degree of freedom of this cosmology is $\chi_{\text {dof }}^{2}=655.33 / 1598=0.41$.

To facilitate a direct comparison between $R_{\mathrm{h}}=c t$ and $\Lambda \mathrm{CDM}$, we show in Figure 4 the best-fitting theoretical curves for the concordance flat $\Lambda \mathrm{CDM}$ model with fixed $\Omega_{\mathrm{m}}=0.3$ (dashed), the optimized flat $\Lambda \mathrm{CDM}$ model with $\Omega_{\mathrm{m}}=$ $0.582_{-0.059}^{+0.074}$ (dotted-dashed), and the $R_{\mathrm{h}}=c t$ universe (solid). On the basis of their $\chi_{\text {dof }}^{2}$ values, the optimized $\Lambda$ CDM model 
and the $R_{\mathrm{h}}=c t$ universe appear to fit the data comparably well. Because these models have different numbers of free parameters, however, a simple $\chi_{\text {dof }}^{2}$-minimization is not sufficient to fairly judge which is a better match to the data. A comparison of the likelihoods indicating which cosmology is closest to the "true" model must be based on model selection criteria, which we discuss next.

\subsection{Statistical Performance with Quasars}

Since the sample we have is very large, we apply the most appropriate model selection tool, the Bayes Information Criterion (BIC; Schwarz 1978), to test the statistical performance of the models:

$$
\mathrm{BIC}=\chi^{2}+(\ln N) f,
$$

where $N$ is the number of data points and $f$ is the number of free parameters. The BIC is a large-sample $(N \rightarrow \infty)$ approximation to the outcome of a conventional Bayesian inference procedure for deciding between models. Among the models being tested, the one with the least BIC score is the one most preferred by this criterion. A more quantitative ranking of models can be computed as follows. With $\mathrm{BIC}_{\alpha}$ characterizing model $\alpha$, the unnormalized confidence that this model is correct is the "Bayes weight" $\exp \left(-\mathrm{BIC}_{\alpha} / 2\right)$. The relative likelihood of model $\alpha$ being correct is then

$$
P(\alpha)=\frac{\exp \left(-\mathrm{BIC}_{\alpha} / 2\right)}{\sum_{i} \exp \left(-\mathrm{BIC}_{i} / 2\right)},
$$

where the sum in the denominator runs over all of the models being tested simultaneously. The outcome of this analysis is summarized in Table 1. According to these results, we conclude that $R_{\mathrm{h}}=c t$ is slightly preferred over $\Lambda$ CDM with a likelihood of $\sim 57.69 \%$ versus $42.31 \%$. The concordance model with a fixed $\Omega_{\mathrm{m}}=0.3$ can be safely discarded as having a probability of only $\sim 10^{-5}$ of being correct compared to the other two fits reported here. To facilitate the comparison, Table 1 also shows the individual BIC values and each model's relative likelihood.

\section{Summary and Discussion}

In this work, we have proposed a method for calibrating the luminosity distance in a model-independent way, and using this to measure the spatial curvature. This approach is achieved by combining observations of quasars and cosmic chronometers. First, we use the discrete cosmic-chronometer measurements to reconstruct the continuous Hubble function $H(z)$ using a polynomial fit. The co-moving distance function can then be derived by directly calculating the integral of the reconstructed $H(z)$ function. With the curvature parameter $\Omega_{K}$ taken into account, we can transform the co-moving distance into the curvature-dependent luminosity distance $D_{L}^{\text {cal }}\left(\Omega_{K}, z\right)$. Finally, based on the X-ray versus UV luminosity correlation for quasars in the redshift range overlapping with $H(z)$, we combine the redshift and flux measurements of 1330 sources at $z<2$ with $D_{L}^{\text {cal }}\left(\Omega_{K}, z\right)$ to constrain both the curvature parameter and the parameters characterizing the luminosity relation in a model-independent way.
Table 2

Best-fitting Results in Different Cosmological Models using only the 1330 Quasars at $z<2$

\begin{tabular}{lcccc}
\hline \hline Model & $\Omega_{\mathrm{m}}$ & $\chi_{\text {dof }}^{2}$ & BIC & Likelihood \\
\hline Concordance & 0.3 (fixed) & 0.43 & 568.62 & $0.02 \%$ \\
$\Lambda$ CDM & $0.580_{-0.068}^{+0.087}$ & 0.41 & 556.18 & $10.15 \%$ \\
$R_{\mathrm{h}}=c t$ & $\ldots$ & 0.41 & 551.82 & $89.83 \%$ \\
\hline
\end{tabular}

This analysis suggests that the curvature parameter is constrained to be $\Omega_{K}=-0.918 \pm 0.429$, which deviates slightly from zero. That is, the current quasar data appear to favor a mildly closed universe at the $2.1 \sigma$ level. The optimized correlation parameters are $\gamma=0.612 \pm 0.017, \kappa^{\prime}=$ $7.447 \pm 0.500$, and $\sigma_{\text {int }}=0.233 \pm 0.005$. Assuming a standard flat $\Lambda$ CDM model with $\Omega_{\mathrm{m}}=0.3$, Risaliti \& Lusso (2019) found the optimized values of the correlation parameters to be $\gamma=0.633 \pm 0.002$ and $\sigma_{\text {int }}=0.24$. Our constraints are very similar to those of Risaliti \& Lusso (2019), though not exactly the same. This comparison between the two approaches attests to the reliability of our calculation, but also indicates the importance of developing a cosmology-free calibration.

Assuming that the extrapolation of the calibrated luminosity relation beyond $z \sim 2$ is valid, we obtained a new sample of distance moduli for 1598 different quasars, and used them to compare two competing cosmological scenarios, i.e., $\Lambda \mathrm{CDM}$ and the $R_{\mathrm{h}}=c t$ universe. We showed that the latter fits the data with a reduced $\chi_{\text {dof }}^{2}=655.33 / 1598=0.41$. By comparison, the optimal flat $\Lambda \mathrm{CDM}$ model fits these same data with a reduced $\chi_{\text {dof }}^{2}=648.57 / 1597=0.41$ for a matter density parameter $\Omega_{\mathrm{m}}=0.582_{-0.059}^{+0.074}$. The model comparison shows that $R_{\mathrm{h}}=c t$ is slightly preferred over $\Lambda \mathrm{CDM}$ with a likelihood of $\sim 57.7 \%$ versus $42.3 \%$ when $\Omega_{\mathrm{m}}$ is allowed to deviate from its concordance value. $R_{\mathrm{h}}=c t$ is much more strongly preferred over $\Lambda$ CDM, however, when the latter is based on the concordance parameter values.

To examine whether the calibrated luminosity relation can reliably be extrapolated to high $z$, we also used just the restricted sample of 1330 calibrated quasar distance moduli at $z<2$ to compare different cosmological models. The results are shown in Table 2 . The likelihoods indeed change somewhat, but not qualitatively. $R_{\mathrm{h}}=c t$ is more strongly favored over $\Lambda$ CDM with a likelihood of $\sim 89.8 \%$ versus $10.2 \%$. The outcomes based on the reduced and complete samples are therefore consistent with each other.

We are grateful to the anonymous referee for useful comments that have helped us improve the presentation of the paper. This work is partially supported by the National Natural Science Foundation of China (grant Nos. 11603076, 11673068, 11725314, and U1831122), the Youth Innovation Promotion Association (2017366), the Key Research Program of Frontier Sciences (grant Nos. QYZDB-SSW-SYS005 and ZDBS-LY-7014), the Strategic Priority Research Program "Multi-waveband gravitational wave universe" (grant No. XDB23000000) of Chinese Academy of Sciences, the "333 Project" of Jiangsu Province, and the Guangxi Key Laboratory for Relativistic Astrophysics.

\section{ORCID iDs}

Jun-Jie Wei (iD https://orcid.org/0000-0003-0162-2488 Fulvio Melia (i) https://orcid.org/0000-0002-8014-0593 


\section{References}

Amati, L., D’Agostino, R., Luongo, O., Muccino, M., \& Tantalo, M. 2019, MNRAS, 486, L46

Aubourg, É., Bailey, S., Bautista, J. E., et al. 2015, PhRvD, 92, 123516

Avni, Y., \& Tananbaum, H. 1986, ApJ, 305, 83

Bañados, E., Venemans, B. P., Mazzucchelli, C., et al. 2018, Natur, 553, 473

Bernstein, G. 2006, ApJ, 637, 598

Cai, R.-G., Guo, Z.-K., \& Yang, T. 2016, PhRvD, 93, 043517

Cao, S., Qi, J., Biesiada, M., et al. 2019, PDU, 24, 100274

Clarkson, C., Cortês, M., \& Bassett, B. 2007, JCAP, 8, 011

Denissenya, M., Linder, E. V., \& Shafieloo, A. 2018, JCAP, 3, 041

Eisenstein, D. J., Zehavi, I., Hogg, D. W., et al. 2005, ApJ, 633, 560

Foreman-Mackey, D., Hogg, D. W., Lang, D., \& Goodman, J. 2013, PASP, 125,306

Gong, Y., \& Wang, A. 2007, PhRvD, 75, 043520

Hogg, D. W. 1999, arXiv:astro-ph/9905116

Ichikawa, K., Kawasaki, M., Sekiguchi, T., \& Takahashi, T. 2006, JCAP, 12,005

Jimenez, R., \& Loeb, A. 2002, ApJ, 573, 37

Khadka, N., \& Ratra, B. 2019, arXiv:1909.01400

L'Huillier, B., \& Shafieloo, A. 2017, JCAP, 1, 015

Li, Y.-L., Li, S.-Y., Zhang, T.-J., \& Li, T.-P. 2014, ApJL, 789, L15

Li, Z., Ding, X., Wang, G.-J., Liao, K., \& Zhu, Z.-H. 2018, ApJ, 854, 146

Li, Z., Wang, G.-J., Liao, K., \& Zhu, Z.-H. 2016, ApJ, 833, 240

Liao, K., Li, Z., Wang, G.-J., \& Fan, X.-L. 2017, ApJ, 839, 70

López-Corredoira, M., Melia, F., Lusso, E., \& Risaliti, G. 2016, IJMPD, 25, 1650060

Lusso, E., Piedipalumbo, E., Risaliti, G., et al. 2019, A\&A, 628, L4

Lusso, E., \& Risaliti, G. 2016, ApJ, 819, 154

Lusso, E., \& Risaliti, G. 2017, A\&A, 602, A79

Melia, F. 2003, The Edge of Infinity, Supermassive Black Holes in the Universe (Cambridge: Cambridge Univ. Press)

Melia, F. 2007, MNRAS, 382, 1917
Melia, F. 2013, A\&A, 553, A76

Melia, F. 2019, MNRAS, 489, 517

Melia, F., \& Shevchuk, A. S. H. 2012, MNRAS, 419, 2579

Mortlock, D. J., Warren, S. J., Venemans, B. P., et al. 2011, Natur, 474, 616

Mortsell, E., \& Jonsson, J. 2011, arXiv:1102.4485

Ooba, J., Ratra, B., \& Sugiyama, N. 2018a, ApJ, 864, 80

Ooba, J., Ratra, B., \& Sugiyama, N. 2018b, ApJ, 866, 68

Planck Collaboration, Aghanim, N., Akrami, Y., et al. 2018, arXiv:1807.06209

Qi, J.-Z., Cao, S., Zhang, S., et al. 2019, MNRAS, 483, 1104

Rana, A., Jain, D., Mahajan, S., \& Mukherjee, A. 2017, JCAP, 3, 028

Räsänen, S., Bolejko, K., \& Finoguenov, A. 2015, PhRvL, 115, 101301

Riess, A. G., Casertano, S., Yuan, W., Macri, L. M., \& Scolnic, D. 2019, ApJ, 876,85

Risaliti, G., \& Lusso, E. 2015, ApJ, 815, 33

Risaliti, G., \& Lusso, E. 2019, NatAs, 3, 272

Ruan, C.-Z., Melia, F., Chen, Y., \& Zhang, T.-J. 2019, ApJ, 881, 137

Sapone, D., Majerotto, E., \& Nesseris, S. 2014, PhRvD, 90, 023012

Schwarz, G. 1978, AnSta, 6, 461

Scolnic, D. M., Jones, D. O., Rest, A., et al. 2018, ApJ, 859, 101

Shafieloo, A., \& Clarkson, C. 2010, PhRvD, 81, 083537

Tegmark, M., Eisenstein, D. J., Strauss, M. A., et al. 2006, PhRvD, 74, 123507

Virey, J.-M., Talon-Esmieu, D., Ealet, A., Taxil, P., \& Tilquin, A. 2008, JCAP, 12,008

Wang, G.-J., Wei, J.-J., Li, Z.-X., Xia, J.-Q., \& Zhu, Z.-H. 2017, ApJ, 847, 45

Wei, J.-J. 2018, ApJ, 868, 29

Wei, J.-J., Melia, F., \& Wu, X.-F. 2015, AJ, 149, 102

Wei, J.-J., \& Wu, X.-F. 2017, ApJ, 838, 160

Wright, E. L. 2007, ApJ, 664, 633

Wu, X.-B., Wang, F., Fan, X., et al. 2015, Natur, 518, 512

Xia, J.-Q., Yu, H., Wang, G.-J., et al. 2017, ApJ, 834, 75

Yu, H., Ratra, B., \& Wang, F.-Y. 2018, ApJ, 856, 3

Yu, H., \& Wang, F. Y. 2016, ApJ, 828, 85

Zhao, G.-B., Xia, J.-Q., Li, H., et al. 2007, PhLB, 648, 8 